\title{
Register rerum ad Vol. 140
}

\section{Confecit G. Boehm, Basel}

(B) = Buchbesprechungen - Livres nouveaux - Book reviews $(\mathrm{R})=$ Referate von Vorträgen, die an anderer Stelle ausführlich erschienen sind, oder erscheinen werden. Einzelheiten wurden in diesem Register rerum nicht berücksichtigt. - Abstract

$\mathrm{s}$ of lectures that have been (or will be) published in extenso elsewhere. Details are not been included in this Register rerum. -Rapports de conferences qui ont été (ou seront) publiées ailleurs in extenso. Ce Register rerum ne contient pas de details.

Abflußkoeffizient des Kammer-wassers, v. «Suction cup»

Abflußwiderstand des Rammer-wassers, v. Trabeculum

Ablatio retinae; prophylaktische Be-handlung der Netzhautablösung, 288 (B)

-; v. Fluoreszenz, Linsentrübungen, Macula, Netzhautabhebung, Retinal detachment, Senile macular degeneration

Accommodation, v. Empty visual field

ACTH (= adrenocorticotropes Hor-mon == «Exacthin»), v. Netzhaut-zirkulationsstörungen

Adaptation à Tobscurité,

v. Adaptation, Double-flash test

Adaption, pupillomotorial, of the eye, 379

Adénosintriphosphate, $v$. Retina

Aderhaut; Untersuchungen über die Gewebsmastzellen bei Melano-blastom der Aderhaut, 41

Adrenocorticotropes Hormon

$(=\mathrm{ACTH}=$ «Exacthin»), v. Netz-hautzirkulationsstörungen

Advances, v. Progrès

Affen, v. Primaten

Agar-electrophoresis, $\mathrm{v}$.Lens proteins

Ageing, v. Gerontoxon, «Suction cup»

Akkommodation, v. Empty visual field

Alpha-chymotrypsin in cataract surgery, "enzymatic zonulolysis", 22

; histological picture of the human eye operated upon by fermentative zonulolysis, 251

Altern, v. Gerontoxon, «Suction cup»

Amblyopie, v. Schielamblyopie, Sehbehinderung

Amotio retinae, $v$. Ablatio retinae

Angle of anterior chamber, v. Trabeculum

Angle iridio-cornéen, v. Trabeculum

Animals, domestic, anatomy and

histology of the eye and orbit,

409 (B) Année thérapeutique et clinique en

ophtalmologie (Vol. 10), 352 (B) Anomalien des stereoskopischen Se-

hens bei Schielenden und ihre Be-

handlung, 158 (B) Antibiotica et Chemotherapia 
(Vol. 7), 80 (B) -- (Vol. 8), 222 (B) Anticoagulantien; Behandlung der retinalen Venenthrombose mit Anticoagulantien, 225 -; v. Senile macular degeneration APC (= Adenoidal-Pharyngeal-Conjunctival)-Virus-Gruppe, v. Keratoconjunctivitis epidemica Aqueous humour, v. Fluoreszenz, Inflammations Aqueous humour flow coefficient, v. Coefficient. .. Aqueous-trabecular barrier, v. Trabeculum Arcus senilis, v. Gerontoxon Arteriosclerosis, v. Senile macular degeneration AT 10, v. Auge und Tetanie Atlas of diabetic retinopathy; a photographic study (Acta ophthalmologica, Suppl. 55), 156 (B) ATP (Adénosintriphosphate), v. Retina Auge und Tetanie, 159 (B) Augenbinnendruck, v. HarnstoffInfusion, «Suction cup» Augenheilkunde und Gewebsmechanik, 159 (B) Augenheilkunde, Lehrbuch (3. Aufl.), 283 (B) Augenhöhle, v. Orbita Augenlid, v. Narbenentropium Augenveränderungen bei Xeroderma pigmentosum, v. Ocular changes Augen, v. Animals, Eye(s), Virus 412 Register rt Autoradiographische Studien zur Jodanreicherung im Auge durch Iontophorese, 44 Aveugle, v. Blindheit, Blindness Bacille Calmette-Guérin (BCG), v. BCG vaccination Barcelona, v. Instituto Barraquer Barraquer, v. Instituto Barraquer Barrière humeur aqueuse-collagène, $v$. Trabeculum Basophile Leukozyten, v. Gewebs-mastzellen

Bâtonnets de la rétine humaine, etude au microscope électronique, 200

BCG vaccination; eye disease following BCG vaccination (Acta oph-thalmologica, Suppl. 57), 157 (B)

Beef, v. Retina

Behandlung, v. Prophylaxis, Therapie

Bestrahlung, v. Pterygium

Beta-radiation; prevention of recurrent pterygium by ${ }^{\wedge}{ }^{\wedge}$-radiation, 369

Bindegewebe, vererbbare Störungen (Heritable disorders of connective tissue) (Deutsche Übersetzung), 80 (B)

-, v. Orbita

Bindehaut; Beiträge zu den Virus-erkrankungen der Bindehaut (Mol-luscum contagiosum), 241

-; v. Conjunctival fluid

Binocular imbalance, deviations, statistics, 180

Biochemical and biophysical behaviour of the trabeculum, 120

Biomikroskopie, v. Photographie

Biophysical and biochemical behavior of the trabeculum, 120

Blindheidsoorzaken in Nederland (The causes of blindness in the Netherlands), 351 (B)

Blindheit, kongenitale, des Neu-geborenen, Diagnose, v. Cécité

Blindheit, Sehbehinderung und ihre Rehabilitation, 287 (B)

Blindheit, v. Blindness

Blindness, congenital, of the newborn, diagnosis, v. Cécité 
Blindness in the Netherlands, causes (Blindheidsoorzaken in Nederland), 351 (B)

ad Vol. 140

Blindness, v. Blindheit

Blood, v. Mastzellen, Sickle-cell

retinopathy Blood circulation, v. Netzhautzirku-

lationsstörungen Blood plasma (electrophoretic

picture), v. Lens proteins Bceuf, v. Retina Book reviews, 79-80 (B); 156-159 (B);

222 (B); 283-288 (B); 351-352 (B);

409-410 (B) Dovís, v. Retina Buchbesprechungen, 79-80 (B); 156

-159 (B); 222 (B); 283-288 (B);

351-352 (B); 409-410 (B)

$\mathrm{Ca}, \mathrm{v}$. Tetanie

Caecitas, v. Blindheit, Blindness Calcium-Stoffwechsel, v. Tetanie Capsule cristallinienne, v.

Linsen-

trübungen Cataract; photocoagulative operation

of cataract and secondary

cataract, 303 Cataract operation; histological picture of the human eye operated

upon by fermentative zonulolysis,

251 Cataract surgery; alpha-chymotryp-

sin in cataract surgery. "Enzymatic

zonulolysis”, 22 -;v. Tetanie

Cattle, horned cattle, v. Retina Cécité; Гintérêt de Гélectrorétino-

graphie dans le diagnostic de la

cécité du nouveau-né, 1 -;v. Blindheit, Blindness Cellular reactive proteins (CRP); the

significance of C-reactive protein

determinations in inflammatory

conditions of the eye, 104 Central retinal vein thrombosis,

v. Netzhautzirkulationsstörungen,

Venenthrombose Central vein thrombosis, v. Netzhautzirkulationsstörungen Champ visuel vide,

v. Empty visual

field Chemotherapia et Antibiotica

(Vol. 7), 80 (B) -- (Vol. 8), 222 (B) Chibret; médaille d'or Chibret (1961)

(Ligue contre le trachome), 224

Register rerum ad Vol. 140

413

-;prix Chibret (1961), 224

Chirurgie, v. Eye surgery, Goniotripsy

Chorioidea, v. Melanoblastom

$\alpha$-Chymotrypsin, v. Alpha-chymo-trypsin

Cicatrix, v. Narbenentropium

Clinical, v. Conjunctival fluid

Clinique; Гannée thérapeutique et clinique en ophtalmologie (Vol. 10), 352 (B)

-; v. Klinik

Coagulation, diathermy coagulation, v. Linsentrübungen 
Coefficient de debit de Thumeur aqueuse, modifications, en function de $\Gamma$ âge, calculé au moyen de la «suction cup», 215

Coefficient de la rigidité, v. Rigiditätskoeffizient

Collagen, trabecular collagen; biochemical and biophysical behavior of the trabeculum, 120

Colour perception, v. Empty visual field

Coming medical meetings, 160, 224, 410

Complications in eye surgery, management, 157 (B)

Concilium Ophthalmologicum Uni-versale, congressus (1960), 159

Concours, v. Instituto BaГraquer

Congenital blindness, diagnosis, v. Cécité

Congenital glaucoma; goniotripsy: an operation for congenital glaucoma, 14

Congress; XIXth International Congress of Ophthalmology (New Delhi, 1962), 288

-; Ophthalmological Congress of Japan, 64th, annual (1960), 335-343 (R)

-;v. Concilium, Kongreßkalender

Congress, 224

Conjunctiva; Beiträge zu den Virus-erkrankungen der Bindehaut (Molluscum contagiosum), 241

-; cysticerque sous-conjonctival du cul-de-sac inférieur, 237

,- v. Zystizercus

Conjunctival fluid, cytology (Experimental and clinical studies based

on a quantitative pipette method) (Acta ophthalmologica, Suppl. 59), 158 (B)

Conjonyctives, structures conjunctives, v. Orbita

Conjunctivitis, v. Keratoconjuncti-vitis epidemica

Connective tissue, v. Orbita

Connective tissue (heritable disorders), v. Bindegewebe

Conseil International d'Ophtal-mologie, reunion (1960), 159

Convalescent patients, serum, v. Keratoconjunctivitis epidemica

Cornea; Infiltrat der Hornhaut im Gebiete des Gerontoxons, 161

-; a rare form of keratitis, Isolation of a virus, 87

Cornea, v. Iontophorese, Keratoconjunctivitis epidemica

Corneal ulcer, rodent (Mooren), possibility of a virus etiology, 311

Corpus adiposum orbitae, v. Coussi-net adipeux de Torbite

Corticosteroide, v. Chemotherapia, Nebennieren-Corticoide

Coussinet adipeux de Torbite; les structures conjunctives et le coussinet graisseux orbitaire (Acta anatomica, Suppl. $39=1$ ad Vol. 41), 287 (B)

Cow, v. Retina

$\mathrm{C}$ (= cellular)-reactive proteins; the significance of C-reactive protein determinations in

inflammatory conditions of the eye, 104

Cristallin, v. Jodanreicherung, Lens proteins, Linsentrübungen

CRP (= Cellular reactive protein), v. Inflammations

Cul de sac conjonctival, v. Cysticerque

Cysticercus cellulosae, v. Cysticerque, Zystizercus

Cysticerque sous-conjonctival du cul-de-sac inférieur, 237

-, v. Zystizercus

Cytology of conjunctival fluid

(Experimental and clinical studies based on a quantitative pipette method (Acta ophthalmologica, Suppl. 59), 158 (B) 


\section{Register $r \varnothing$}

Dark adaptation, v. Adaptation,

Double-flash test Debit de Thumeur aqueuse, coefficient, v. Coefficient... Décollement de la rétine et trou dans

la macula, 94 Décollement de la rétine, v. Ablatio

retinae Dégénérescence maculaire senile,

v. Senile macular degeneration Demi-singes, v. Primaten Detachment of the retina,

v. Ablatio retinae Deutsche Ophthalmologische Gesell-

schaft, 62. Zusammenkunft (1959),

343-350, 401-408 (R) Deviations of binocular imbalance,

statistics, 180 Diabetic retinopathy, atlas; a photographic study (Acta ophthalmo-

logica, Suppl. 55), 156 (B) Diabetic retinopathy (An ophthalmo-

scopic study with a discussion of

the morphologic changes and the

pathogenic factors in this disease),

409 (B) Diagnose; Differential-Diagnose und

Therapie der Gesichtsneuralgien,

351 (B) -; Гintérêt de Гélectrorétinographie

dans le diagnostic de la cécité du

nouveau-né, 1 -; Vom Symptom zur Diagnose

(Lehrbuch der medizinischen

Symptomatologie), 79 (B) Diathermy coagulation, v. Linsen-

trübungen Dicumarolpräparat, v. Venen-

thrombose Differential-Diagnose und Therapie

der Gesichtsneuralgien, 351 (B) Diskontinuitätszonen, optische,

v. Photographie Domestic animals, anatomy and

histology of the eye and orbit,

409 (B) Doppelreize, v. Double flash test Double-flash test; a contribution to

the physiology of the electro-

retinographic double-flash test, 267 -; v. Stimulations couplées Druck, intraokularer, v. Intra-

okularer Druck

ad Vol. 140

Drucksenkung, v. Harnstoff-

Infusion Dunkeladaptation, v. Adaptation,

Double-flash test

Ecoulement de Thumeur aqueuse,

v. «Suction cup» Einschlußkörperchen, v. Keratitis,

Ulcus rodens Elastizität, v. Rigiditätskoeffizient Electro-coagulation, v. Elektro-

koagulation Electronique, microscope -; etude

des bâtonnets de la rétine humaine

au microscope electronique, 200 Electrophoretic picture,

v. Lens proteins Electroretinography; a contribution

to the physiology of the electrore-

tinographic double-flash test, 267 -; Гintérêt de Гélectrorétinographie

dans le diagnostic de la cécité du

nouveau-né, 1 -; la réponse électro-rétínographique 
aux stimulations couplées chez les

sujets normaux, 353 Electro ..., v. Elektro ... Elektrokoagulationen bei Netzhaut-

ablösungen, Linsentrübungen, 98 Elektro . . ., v. Electro . .. Empty visual field, vision, 322

Endocular liquids, v. Fluoreszenz Entropium; ein Beitrag zur operati-

ven Lösung des Narbenentropiums,

168 Entzündungen des Auges, Einfluß

der Nebennieren-Corticoide,

287 (B)

, v. Inflammations

Enucleatio bulbi; zur einfachen

Stumpfbildung bei Enucleatio und

Evisceratio bulbi, 172 "Enzymatic zonulolysis"; alpha-chy-

motrypsin in cataract surgery, 22

; v. Zonulolysis

Epidemic keratoconjunctivitis,

v. Keratoconjunctivitis epidemica Erythrocytes, v. Sickle-cell retinopathy Espagne, v. Instituto

Barraquer Evisceratio bulbi; zur einfachen

Stumpfbildung bei Enucleatio und

Evisceratio bulbi, 172

Register reruin

«Exacthin» (Sandoz) (= AGTH),

v. Netzhautzirkulationsstörungen Exzent $\Gamma$ izität de $\Gamma$ monokularen Fixation, v. Schielamblyopie Eye, changes (Xeroderma pigmento-

sum), v. Ocular changes Eye disease following BCG vaccination (Acta ophthalmologica, Suppl. 57), 157 (B) Eye lens proteins, v. Lens proteins Eyelid, v. Narbenentropium Eye surgery, management of complications, 157 (B) Eyes; you and your eyes, 352 (B) Eyes, v. Domestic animals, Virus

Face; syndrome douloureux de la face, v. Gesichtsneuralgien

Facial neuralgias, v. Gesichtsneuralgien

Faibles de vue, v. Sehbehinderung

Farbwahrnehmung, v. Empty visual field

Fat, tissue, v. Orbita

Feinstruktur, v. Bâtonnets

Fermentative zonulolysis; histologi-cal picture of the human eye operated upon by fermentative zonulolysis, 251

-; "enzymatic zonulolysis"; alpha-chymotrypsin in cataract surgery, 22

Ferments, v. Fermentative ... ., Retina

Fettgewebe in der Orbita, v. Orbita

Fever therapy, v. Inflammations

Fiches électromécaniques, v. Strabismus

Fiebertherapie, v. Inflammations

Fixation, monokulare; über die In-stabilität und Exzentrizität der monokularen Fixation bei

Schielamblyopie, 81

Flügelfell, v. Pterygium

Flüssigkeiten im Auge, v. Fluoreszenz 
Fluoreszenz der Flüssigkeiten im Auge, weitere quantitative spektro-photometrische

Bemerkungen, 115

Fornix conjunctival, v. Cysticerque

Fortschritte, v. Progrès

Gefäße, v. Netzhautzirkulationsstörungen, Thrombose Genetik, v. Strabismus

ad Vol. $140 \quad 415$

Gerontoxon; Infiltrat der Hornhaut im Gebiete des Gerontoxons, 161

Gesellschaftsberichte, 280-283 (R); 335-343 (R); 343-350, 401-408 (R)

Gesichtsfeld, leeres, v. Empty visual field

Gesichtsneuralgien, Differential-Diagnose und Therapie, 351 (B)

Gewebsmastzellen; Untersuchungen über die Gewebsmastzellen des menschlichen Auges bei

Melano-blastom der Aderhaut, 41

Gewebsmechanik und Augenheilkun-de, 159 (B)

Glandulae parathyreoideae, v. Tetanie

Glandulae suprarenales, v. Neben-nieren-Corticoide

Glaucoma, congenital; goniotripsy: an operation for congenital glaucoma, 14

Glaucomtherapie,Harnstoff-Infusion, 135

Glaucoma, v. Trabeculum

Glaukom (Ein Handbuch), 286 (B)

Glaukom, v. Glaucoma, Glaucom-therapie, "Suction cup"

Glucose, v. Retina

Goniotripsy; an operation for congenital glaucoma, 14

Graisseux, coussinet graisseux, v. Orbita

Grauer Star, v. Cataract

Greisenbogen, v. Gerontoxon

Halbaffen, v. Primaten Half-monkeys, v. Primaten Handbook of physiology Section I:

Neurophysiology (volume II),

156 (B) Handbuch, v. Glaukom Harnstoff-Infusion in der Glaucom-

therapie, 135 Haustiere, v. Domestic animals "Heparinoid" (Roche), v. Senile

macular degeneration Heredopathy, v. Bindegewebe Hexokinase, v. Hexoquinasique ... .

Hexoquinasique, activité -; à propos

de $\Gamma$ activité hexoquinasique de la

rétine du bceuf, 193 Histological picture of the human eye

operated upon by fermentative

zonulolysis, 251

416 Register $\mathrm{r}<$

Holes of the macula, v. Macula

Hornhaut; Infiltrat der Hornhaut im Gebiete des Gerontoxons, 161

Hornhaut, v. Cornea, Corneal ulcer, Keratokonjunctivitis epidemica

Hornhautentzündung, v. Keratitis

Humaine, rétine humaine ; etude des bâtonnets de la rétine humaine au microscope électronique, 200

Humeur aqueuse, v. Fluoreszenz, Inflammations

Humeur aqueuse, coefficient de debit, v. "Suction cup"

«Hydergin» (Sandoz), v. Netzhaut-zirkulationsstörungen

Hypophysis, v. Pituitary tumors 
I181, v. Jodanreicherung

Immunisation; the influence of immunisation against eye lens protein on the electrophoretic picture of blood plasma and eye lens proteins (Preliminary communication), 333

Implantat (Nylonimplantat), v. Enucleatio bulbi

Inclusion bodies, v. Keratitis, Ulcus rodens

Indien $<, v$. International Congress

Infiltrat der Hornhaut im Gebiete des Gerontoxons, 161

Inflammations; the significance of C-reactive protein determinations in inflammatory conditions of the eye, 104

-; v. Nebennieren-Corticoide

Instabilität und Exzentrizität der monokularen Fixation bei Schiel-amblyopie, 81

Instituto Barraquer, dritter inter-nationaler Kurs in Ophthalmolo-gie, (1961), 223

-, Concours de travaux ophtalmolo-giques, 223

International Congress of Ophthalmology, XIXth (New Delhi), 288

Internationaler Kurs in Ophthalmo-logie, v. Instituto Barraquer

Intraokularer Druck; Abhängigkeit des Rigiditätskoeffizienten von der Höhe des intraokularen

Drucks, 55

-;v. Harnstoff-Infusion, «Suction cup»

ad Vol. 140

Iontophorese; autoradiographische Studien zur Jodanreicherung im Auge durch Iontophorese, 44

Iris-Struktur der Primaten, 27

Irradiation, v. Pterygium

Isotope, radioaktive, v. Beta-radiation, Jodanreicherung

Japan; Ophthalmological Society of Japan, 64th annual, congress (1960), 335-343 (R)

Jodanreicherung im Auge durch Iontophorese, autoradiographische Studien, 44

Jod, radioaktives, v. Jodanreicherung

Kalzium-Stoffwechsel, v. Tetanie Kammerwasser, v. Fluoreszenz,

Inflammations, Trabeculum Kammerwasser, Abflußkoeffizient,

v. «Suction cup» Kammerwinkel, v. Trabeculum Kaninchen, v. Fluoreszenz,

Iontophorese Katarakt, v. Cataract Kataraktoperation, v. Cataract

surgery, Zonulolysis Keratitis, a rare form. Isolation of a

virus, 87 Keratoconjunctivitis epidemica, ein

Beitrag zur Therapie durch lokale

Anwendung von Reconvalescenten-

serum, 296 Kinderheilkunde, v. Pédiatrie Klinik und Therapie der Nebenwir-

kungen, 410 (B) Klinik, v. Clinique, Conjunctival

fluid Klinische Neuroradiologie, 285 (B) Kollagen (der Trabekeln im Kammerwinkel), v.

Collagen Kongenitale Blindheit, Diagnose,

v. Cécité Kongenitales Glaukom, v. Glaucoma Kongreß, v. Concilium Kongreßkalender, 160, 224, 410 Kurs, v. Instituto Barraquer

Lampe à fente et photographie, 129 Laryngologie, v. Oto- .. . Lapin, v. Fluoreszenz,

Iontophorese Leeres Gesichtsfeld, v. Empty visual field

Register Terum ad Vol. 140

417

Lehrbuch der Augenheilkunde (3. Aufl.), 283 (B) 
Lens proteins; the influence of immunisation against eye lens protein on the electrophoretic picture of blood plasma and eye lens proteins (Preliminary communication), 333

Lens, v. Iontophorese, Linsentrübungen

Leucocytes, basophiles, v. Gewebsmastzellen

Lichtkoagulation, v. Cataract

Lid, v. Narbenentropium

Life-long cures and improvements after transsphenoidal operation of pituitary tumors (Acta ophthal-mologica, Suppl. 56), 156 (B)

Linsenkapsel, v. Linsentrübungen

Linsentrübungen nach Elektro-koagulationen bei Netzhaut-ablösungen, 98

Liquides endoculaires, v. Fluoreszenz

Livres nouveaux, 79-80 (B); 156-159 (B); 222 (B); 283-288 (B); 351-352 (B); 409-410 (B)

Lochkarten, v. Strabismus

Löcher in der Makula (Makula-schichtlöcher), v. Macula

Macula; trou dans la macula et dé-

collement de la rétine, 94 Macular degeneration, senile, treatment with "Rovigon" and "Heparinoid” (Roche), 147 Macularisse, v. Macula Makula, v. Macula, Macular Man, v. Humaine, rétine humaine Management of complications in eye

surgery, 157 (B) Makuladegeneration, senile, v.

Macular degeneration, senile "Marcumar" (Roche) (= Phenyl-

propyloxycumarin), v. Venen-

thrombose Mastzellen; Untersuchungen über die

Gewebsmastzellen des menschli-

chen Auges bei Melanoblastom der

Aderhaut, 41 Mécanique tissulaire, v. Gewebs-

mechanik Médaille d'or Clibret (Ligue contre

le trachome), 224 Melanoblastom der Aderhaut des

menschlichen Auges, Untersuchungen über die Gewebsmastzellen, 41

Meningitis, tuberculous, ophthalmo-logical aspects (Acta ophthalmolo-gica, Suppl. 61), 409 (B)

Methode, v. Conjunctival fluid, Lam-pe à fente, Operation, Operative ..., «Suction cup»

Microscope électronique ; etude des bâtonnets de la rétine humaine au microscope électronique, 200

Moignon, v. Enucleatio bulbi

Molluscum contagiosum; Beiträge zu den Viruserkrankungen der Binde-haut, 241

Monkeys, v. Primaten

Monokuläre Myopie, v. Myopie monoculaire

Mycobacterium tuberculosis, v. BCC vaccination

Myopie monoculaire; recherches sur la predilection de la myopie monoculaire pour $\Gamma$ ceil droit, 259

Narbenentropium, ein Beitrag zu des-sen operativer Lösung, 168

Nebennieren-Corticoide, Einfluß auf die entzündlichen Reaktionen des Auges, 287 (B)

Nebenwirkungen, Klinik und Thera-pie, 410 (B)

Nerven, v. Gesichtsneuralgien, Neuro...

Netherlands; causes of blindness in Netherlands (Blindheídsoorza-ken in Nederland), 351 (B)

Netherlands Ophthalmological

Society, 146th meeting (1960), 224 
Netzhautabhebung, doppelseitige, durch Zysticercus bedingt, 36

Netzhautablösung; Linsentrübungen nach Elektrokoagulationen bei Netzhautablösungen, 98 -; prophylaktische Behandlung, 288 (B)

-; v. Fluoreszenz, Linsentrübungen, Macula, Macular degeneration, Retinal detachment Netzhautzirkulationsstörungen; Er-fahrungen mit «Panthesin-Hyder-gin» (PH 203) und «Exacthin» (ACTH) bei Netzhautzirkulationsstörungen, 388

Ophlhalmologica, Vol. 140, No. 6 (Dezember 1960)

$1 \gg 1$

Register rerui

418

-; cf. Retina

Neugeborener, kongenitale Blindheit,

Diagnose, v. Cécité Neuralgien; Differential-Diagnose

und Therapie de $\Gamma$ Gesichtsneural-

gien, 351 (B) Neu Oologie, v. Oto-neuro-ophtalmo-

logie Neurophysiology; Handbook of

physiology (section 1, volume II),

156 (B) Neuroradiologie, klinische, 285 (B) New-born, congenital blindness,

diagnosis, v. Cécité New Delhi, v. International Congress Nouveau-né, cécité ; Tintérêt de

Гélectrorétinographie dans le diagnostic de la cécité du nouveau-né,

1 Nylonimplantat, v. Enucleatio bulbi

Ocular changes in Xeroderma pig-mentosum (A case report), 33

Ocular fluids, v. Fluoreszenz

Ocular rigidity, v. "Suction cup"

Oculus, v. Virus

Oculus dexter, v. GEil droit

CEil droit; recherches sur la predilection de la myopie monoculaire pour Гæil droit, 259

CEil, lesions (Xeroderma pigmento-sum), v. Ocular changes

GEil et virus, 286 (B)

CEil, v. Domestic animals, Eye(s)

österreichische Ophthalmologische Gesellschaft, 6. Jahreshauptver-sammlung 1961, 410

Operation; photocoagulative operation of cataract and secondary cataract, 303

-; v. Elektrokoagulationen, Enucleatio bulbi, Eye surgery, Gonio-tripsy, Pituitary tumors, Retinal detachment, Zonulolysis

Operative Lösung des Narbenentro-piums, ein Beitrag, 168

Ophthalmological aspects of tuberculous meningitis (Acta ophthal-mologica, Suppl. 61), 409 (B)

Ophthalmological Congress of Japan, 64th annual (1960), 335-343 (R)

Ophthalmological Society of Netherlands, 146th meeting (1960), 224

ad Vol. 140

Ophthalmological Society of the United Kingdom, 80th annual congress (1960), 280-283 (R)

Ophtalmologie ; Гannée thérapeuti-que et clinique en ophtalmologie (Vol. 10), 352 (B)

-; dritter internationaler Kurs am Instituto Barraquer, 223

mologiques, 223

; Concours de travaux ophtal-

-; Conseil International d'Ophtalmo-logie, reunion (1960), 159 
-;v. Augenheilkunde, Oto-neuro-ophtalmologie

Ophthalmologische Gesellschaft, Deutsche, 62. Zusammenkunft (1959), 343-350, 401-408 (R)

Ophthalmologische Gesellschaft, österreichische, 6. Jahreshaupt-versammlung 1961, 410

Ophthalmologische Gesellschaft, Schweizerische, 53, Jahresver-sammlung (1960), 160

Ophthalmology, XIXth International Congress (New Delhi, 1962), 288

Ophthalmology, year book (1959-1960), 285 (B)

Optical discontinuity, v. Photo-graphie

Orbita ; les structures conjonctives de Torbite et le coussinet grais-seux orbitaire (Acta

anatomica, Suppl. $39=1$ ad Vol. 41), 287 (B)

-; v. Domestic animals

Oryctolagus cuniculus, v. Fluoreszenz, Iontophorese

Osmotherapie, v. Glaucomtherapie

Oto-neuro-ophtalmologie en pédia-trie (Confinia neurologica, Vol. 20, No. 2), 222 (B)

Oto-rhino-laryngologie, progrès (Vol. VI) (Bibliotheca oto-rhino-laryngologica, fasc. 6) 284 (B)

Outflow of the aqueous humour, v. Glaukom, Trabeculum

Pädiaírie, v. Pédiat $\pi$ e Palpebra, v. Narbenentropium < íPanthesin-Hydergin» (PH 203)

(Sandoz), v. Netzhautzirkulations-

störungen Parathyroide, v. Tetanie Paupière, v. Narbenentropium

Register rerum ad Vol. 140

419

Pédiatrie ; Гoto-neuro-ophtalmolo-

gie en pédiatrie (Confinia neurolo-

gica, Vol. 20, No. 2), 222 (B) Perception chromatique, v. Empty

visual field Période réfractaire, v. Double flash

test, Stimulations couplées Phenylpropyl-oxycumarin (= «Mar-cumar», Roche), v. Venenthrom-

bose Phosphate, transfert, v. Retina Phosphorylatión, v. Retina Photobiomicroseopie, v.

Photogra-

phie Photocoagulative operation of cataract and secondary cataract, 303 Photographie ; lampe à

fente et

photographie, 129 -; v. Diabetic retinopathy Physiology of the electroretinogra-

phic double-flash test, a contribution, 267 -, v. Stimulations couplées Physiology, handbook.

Section I:

Neurophysiology (volume II),

156 (B) Pituitary tumors; life-long cures and

improvements' after transsphenoi-

dal operation of pituitary tumors

(Acta ophthalmologica, Suppl. 56),

156 (B) Plasma, blood plasma (electrophore-

tic picture), v. Lens proteins Predilection de la myopie monocu-

laire pour $\Gamma æ i l$ droit, recherches,

259 Predominance in monocular myopia,

v. Myopie monoculaire Pression intravculaire, v. Harnstoff-

Infusion, Rigiditätskoeffizient,

"Suction cup" Pressure, íntra-ocular, v. Harnstoff-

Infusion, Rigiditätskoeffizient, 
"Suction cup" Prevention of recurrent pterygium by

${ }^{\wedge} \wedge$-radiation, 369 Primaten, Iris-Struktur, 27 Progrès en oto-rhino-laryngologie

(Vol. VI) (Bibliotheca oto-rhino-

laryngologica, fasc. 6), 284 (B) Prophylaxis; die prophylaktische

Behandlung der Netzhautablösung.

288 (B)

Protein; the significance of C-reac-tive protein determinations in inflammatory conditions of the eye, 104

-; v. Lensi proteins

Pterygium; prevention of recurrent pterygium by /î-radiation, 369

Punch cards, v. Strabismus

Pupillomotorial adaption of the eye, 379

Pyrétothérapie, v. Inflammations

Quellung des trabekulären Kollagens, v. Trabeculum

Rabbit, v. Fluoreszenz, Iontophorese Radiation; prevention of recurrent

pterygium by $/ \wedge$-radiation, 369 Radioaktive Isotope, $v$. Beta-radiation; Jodanreicherung

Radiographie, v. Autoradiographi-

sche Studien Radiologie, v. Neuroradiologie Reactions sécondaires, v. Neben-

wirkungen Rechtes Auge, v. Myopie monoculaire Recuperation, temps de recuperation,

v. Double flash test, Stimulations

couplées Recurrent pterygium, v. Pterygium Réfractaire, période réfractaire,

v. Double flash test, Stimulations

couplées Regenbogenhaut, v. Iris Rehabilitation; Sehbehinderung,

Blindheit und ihre Rehabilitation,

287 (B) Reizung mit Paaren von Lichtblitzen,

v. Double-flash test, Stimulations

couplées Rekonvalescentenserum, v. Kerato-

conjunctivis epidemica Repetitive light stimuli, v. Double

flash test, Stimulations couplées Resistance to the aqueous humour

outflow, v. Trabeculum Retina ; à propos de Гactivité hexo-

quinasique de la refine du bæuf,

193 -; etude des bâtonnets de la refine

humaine au microscope électroni-

que, 200 -; Erfahrungen mit «Panthesin-

Hydergin» (PH 203) und «Exac-

Register rerui

420

thin» (ACTH) bei Netzhautzirkula-tionsstörungen, 388

-; die prophylaktische Behandlung der Netzhautablösung, 288 (B)

-;v. Macula, Netzhautabhebung, Netzhautablösung, Retinal detachment, Senile macular degeneration

Retinal detachment, surgery, some technical details, 289

-; v. Ablatio retinae

Retinale Venenthrombose, Behandlung mit Anticoagulantien, 225

Retinopathy, sickle-cell retinopathy. Ocular and systematic manifestations of sickle-cell disease (Acta ophthalmologica, Suppl. 58), 156 (B) 
Retinopathia diabetica, v. Diabetic retinopathy

Rhinologie, v. Oto-, ...

Right eye, v. Myopie monoculaire

Rigiditätskoeffizient, Abhängigkeit von der Höhe des intraokularen Drucks ·, 55

Rigidity, ocular rigidity, v. "Suction cup"

Rind, v. Retina

Risse in der Makula, v. Macula

Rodent corneal ulcer, v. Ulcus rodens

Rods, retinal, v. Bâtonnets

«Rovigon» (Roche) (Vitamin A und Vitamin E), v. Senile macular degeneration

Sac conjonctival, v. Conjunctival fluid

Saugnapf, v. Coefficient de debit de Thumeur aqueuse

Scar, v. Narbenentropium

Schielamblyopie; über die Instabili-tät und Exzentrizität der mon-okularen Fixation bei

Schielamblyopie, 81

Schielende; Anomalien des stereo-skopischen Sehens bei Schielenden und ihre Behandlung, 158

(B)

Schielen, v. Strabismus

Schwachsichtigkeit, v. Seh-behinderung

Schweizerische Ophthalmologische Gesellschaft, 53. Jahresversamm-lung (1960), 160

ad Vol. 140

Scleral rigidity coefficient, v. Rigiditätskoeffizient

Scotopic vision, v. Double-flash test

Secondary cataract, v. Photo-coagulative operation

Secondary effects, v. Nebenwirkun-gen

Sehbehinderung, Blindheit und ihre Rehabilitation, 287 (B)

Senile macular degeneration, treatment with "Rovigon" and "Hepa-rinoid" (Roche), 147

Senilität, v. Gerontoxon

Separation of the retina, v. Ablatio retinae

Serum, v. Inflammations

Serum from convalescent patients, v. Keratoconjunctivitis epidemica

Sickle-cell retinopathy; ocular and systematic manifestations of sickle-cell disease (Acta

ophthalmologica, Suppl. 58), 156 (B)

Singes, v. Primaten

Sitzungsberichte, 280-283 (R); 335-343 (R); 343-350, 401-408 (R)

Skotopsie, v. Double-flash test

Slit lamp, v. Photographie

Société Suisse d'Ophtalmologie, assemblée annuelle (1960), 160

Sociétés, comptes rendus, 280-283 (R); 335-343 (R); 343-350, 401-408

(R)

Societies, v. Deutsche Ophthalmologische Gesellschaft, Ophthalmology, Netherlands . .., österreichische ... Society; Japanese Ophthalmological Society, 64th annual congress (1960), 335-343

(R) -; Ophthalmological Society of the United Kingdom, 80th annual congress (1960), 280-283

(R) Society transactions, 280-283 (R); 335-343 (R); 343-350, 401-408 (R) Sous-conjonctival, v. Cysticerque Spain, v. Instituto Barraquer Spaltlampe, v. Photographie Spanien, v. Instituto 
Barraquer Spektrophotometrie; weitere quantitative spektrophotometrische Bemerkungen über die Fluores-zenz der Flüssigkeiten im Auge, 115

Register rerum

Squint, v. Stereoskopisches Sehen, Strabismus

Squint amblyopia, v. Schielamblyopie

Sr90, v. Beta-radiation

Stäbchen, v. Bâtonnets

Star, grauer, v. Cataract

Staroperation, v. Cataract operation, Cataract surgery

Statistics on deviations of binocular imbalance, 180

Statistische Auswertung, v. (Melano-blastom), CEil droit, Rigiditäts-koeffizient, Stimulations couplées, «Suction cup», Venenthrombose

Stereoamblyopie (Stereoamaurose), v. Stereoskopisches Sehen

Stereoskopisches Sehen bei Schielen-den, Anomalien, und ihre Behand-lung, 158 (B)

Steroide, v. Chemotherapia, Neben-nieren-Corticoide

Stimulation by pairs of photic impulses, v. Double-flash test, Stimulations couplées

Stimulations couplées; la réponse électro-rétinographique aux stimulations couplées chez les

sujets normaux, 353

; v. Double flash test

Strabisme, amblyopie avec strabisme, v. Schielamblyopie

Strabisme, v. Stereoskopisches Sehen

Strabismus; statistics on deviations of binocular imbalance, 180

Strontium 90, v. Beta-radiation

Structures conjunctives, v. Orbita

Stump, v. Stumpfbildung

Stumpfbildung, einfache, bei Enucle-atio und Evisceratio bulbi, 172

Submicroscopieal structure, v. Bâtonnets

- Suction cup»; modifications, en fonction de Гâge, du coefficient de debit de Thumeur aqueuse, calculé au moyen de la «suction cup», 215

Surgery; management of complications in eye surgery, 157 (B)

-; some technical details in retinal detachment surgery, 289

-; v. Goniotripsy

Surrénales, glandes -, v. Neben-nieren-Corticoide

ad Vol. $140 \quad 421$

Swelling of the trabecular collagen, v. Trabeculum

Symptom: Vom Symptom zur Diagnose. Lehrbuch der medizinischen Symptomatologie, 79 (B)

Symptomatologie, medizinische, Lehrbuch (Vom Symptom zur Diagnose), 79 (B)

Tableau des congrès, 160, 224, 410

Tarsoplastik, v. Narbenentropium

Tears of the macula, v. Macula

Technical details- in retinal detachment surgery, 289

Temps de recuperation, v. Double flash test, Stimulations couplées

Tension oculaire, v. "Suction cup"

Test, v. Double-flash test, Stimulations couplées

Tetanie und Auge, 159 (B)

Therapie; Гannée thérapeutique et clinique en ophtalmologie (Vol. 10), 352 (B) 
Therapie der Keratoconjunctivitis epidemica durch lokale Anwen-dung von

Reconvalescentenserum, ein Beitrag, 296

Therapie und Klinik der Neben-wirkungen, 410 (B)

Therapie, v. Gesichtsneuralgien, Glaucomtherapie, Inflammations, Pituitary tumors, Prophylaxis, Pterygium, Retinal detachment, Stereoskopisches Sehen, Throm-bose

Thrombose; Behandlung der retina-len Venenthrombose mit Anti-coagulantien, 225

Thrombose (Zentralvenenthrom-bose), v. Netzhautzirkulations-störungen

Tissu conjonctif (lesions héréditai-res), v. Bindegewebe

Tissues, ocular, mechanical properties, v. Gewebsmechanik

Tissulaire, mécanique tissulaire, v. Gewebsmechanik

Tonometrie, v. Rigiditätskoeffizient, "Suction cup"

Trabeculum; biochemical and biophysical behavior of the trabeculum, 120

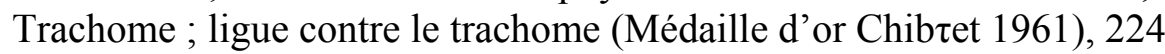

422 Register $\mathrm{r}<$

-; v. Narbenentropium

Transactions, society transactions, 280-283 (R); 335-343 (R); 343-350, 401-408 (R)

Transsphenoidal operation, v. Pituitary tumors

Treatment of senile macular degeneration with "Rovigon" and "Heparinoid" (Roche) 147

Trou dans la macula et décollement de la rétine, 94

Tuberculous meningitis, ophthal-mological aspects (Acta ophthal-mologica Suppl. 61), 409 (B)

Tuberkulose, v. BCG vaccination

Tumoren, v. Melanoblastom, Pituitary tumors

Twin flashes, v, Double flash test, Stimulations couplées

Ulcus rodens; the possibility of a

virus etiology of rodent corneal

ulcer (Mooren), 311 infrastructure, v. Bâtonnets Umschlagsfalte der Conjunctiva,

v. Cysticerque Uniocular myopia, v. Myopie mono-

culaire United Kingdom; Ophthalmological

Society of the United Kingdom,

80th annual congress (1960),

280-283 (R) Urea-treatment (glaucoma), v. Harn-

stoff-Infusion Urée, infusion (glaucome), v. Harn-

stoff-Infusion

Vaccination, v. BCG vaccination Vaisseaux, v. Netzhautzirkulations-

störungen, Thrombose Varia, 159, 223-224, 288 Venenthrombose, retinale, Behand-

lung mit Anticoagulantien, 225 Venenthrombose, v. Netzhautzirku-

lationsstörungen Ventouse, méthode de la ventouse,

v. Coefficient de debit de $\Gamma$ humeur

aqueuse Vererbbare Störungen des Binde-

gewebes (Heritable disorders of

connective tissue), 80 (B) Verm.es, v. Cysticerque, Zystizercus Vers, v. Cysticerque, Zystizercus

Versammlungsberichte, 280-283 (R);

335-343 (R); 343-350, 401-408 (R)

ad Vol. 140

Vessels, v. Netzhautzirkulations-

störungen, Thrombose Vide, champ visuel vide, v. Empty 
visual field Viren $(\mathrm{APC}=$ Adenoidal-Pharyngeal-

Conjunctival-Gruppe), v. Kerato-

conjunctivitis epidemica Virus et ceil, 286 (B) Virus; the possibility of a virus etiology of rodent corneal ulcer

(Mooren), 311 -; a rare form of keratitis. Isolation

of a virus, 87 Viruserkrankungen der Bindehaut,

ein Beitrag (Molluscum eonta-

giosum), 241 Vision in an empty visual field, 322 Vitamin A and vitamin E ("Rovigon"

Roche), v. Senile macular degeneration Vitamin E and vitamin A ("Rovigon"

Roche), v. Senile macular degeneration

Worms, v. Cysticerque, Zystizercus Würmer v. Cysticerque, Zystizercus

Xeroderma pigmentosum, ocular changes (A case report), 33

Year book of ophthalmology (1959-

1960), 285 (B) Yeux, v. Eye(s) You and your eyes, 352 (B)

Zentralvenenthrombose, v. Netzhaut-zirkulationsstörungen, Venenthrombose

Zirkuläres Hornhautinfiltrat, v. Ge-rontoxon

Zirkulationsstörungen, v. Netzhaut-zirkulationsstörungen

Zones de discontinuité optique, v. Photographie

"Zonulolysis, enzymatic"; alpha-chymotrypsin in cataract surgery, 22

; histological picture of the

human eye operated upon by fermentative zonulolysis, 251

Zystizercus; durch Zystizercus be-dingte doppelseitige Netzhaut-abhebung, 36

-.v. -; v. Cysticerque

Zyto ..., v. Cyto ... 